\title{
Conocimientos y actitudes de los universitarios sobre el consumo de cigarrillo y las normas que lo regulan con respecto a sus prácticas, Medellín, 2013*
}

\section{Knowledge and Attitude of University Students on Cigarette Smoking and the Regulations that Rule it in Contrast to their Practices, Medellin, 2013}

\section{Conhecimentos e atitudes dos universitários sobre o consumo de cigarro e normas que regulamentam no que diz respeito a suas práticas, Medellín, 2013}

Fecha de recepción: 03-04-14 Fecha de aceptación: 08-05-14 Disponible en línea: 01-07-14 doi: 10.11144/Javeriana.rgyps13-27.cauc

Cómo citar este artículo:

Patiño-Olarte LM, Zapata-Puerta DM, Olaya-Peláez Á. Conocimientos y actitudes de los universitarios sobre el consumo de cigarrillo y las normas que lo regulan con respecto a sus prácticas, Medellín, 2013. Rev. Gerenc. Polít. Salud. 2014; 13(27): 334-347. http://dx.doi.org/10.11144/Javeriana.rgyps13-27.cauc

\author{
Lina Marcela Patiño-Olarte** \\ Diana Milena Zapata-Puerta*** \\ Álvaro Olaya-Peláez****
}

Artículo de investigación derivado del estudio titulado Conocimientos y actitudes que tienen los estudiantes de una institución universitaria sobre el consumo de cigarrillo y las normas que lo regulan, con respecto a sus prácticas. Medellín, 2013, realizado del 9 de septiembre del 2012 al 8 de julio del 2013, con apoyo de la institución universitaria Tecnológico de Antioquia.

** Profesional en Gerencia de Sistemas de Información en Salud. Editora asistente, Facultad Nacional de Salud Pública, Universidad de Antioquia, Medellín, Colombia. Correo electrónico: linamarce875@gmail.com Correspondencia: Lina Marcela Patiño Olarte, Facultad Nacional de Salud Pública, Universidad de Antioquia, Calle 62 \# 52-59, bloque 33, oficina 228, teléfono: 2196867

*** Profesional en Gerencia de Sistemas de Información en Salud, coordinadora de Aseguramiento y Vigilancia,

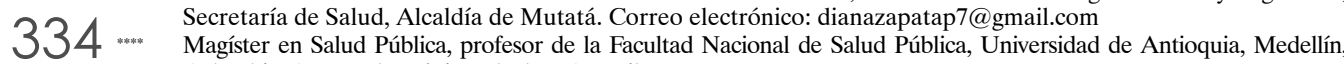
Colombia. Correo electrónico: alvolaya@gmail.com 


\section{Resumen}

Objetivo: describir los conocimientos y actitudes que tienen los estudiantes de una institución universitaria sobre el consumo de cigarrillo y las normas que lo regulan, y cómo estas normas son aplicadas en sus prácticas. Metodología: estudio descriptivo de corte transversal, aplicación de muestreo aleatorio estratificado. Se desarrolló el instrumento tomando algunas variables de las encuestas GATS y GYTS. Resultados y discusión: las mujeres se consideraron fumadoras pasivas (10\%) y los hombres, exfumadores (18,9\%). La prevalencia de vida fue de un 53,3\%, la del último mes $37,7 \%$ en los hombres. El $67,7 \%$ manifestó que las medidas de prevención de la ley colombiana antitabaco no han influido en la disminución del consumo en la sociedad. Se reconoció que existen medidas de prevención de consumo en la institución, pero se considera que se debe fortalecerlas para generar de mayor impacto tanto en la misma institución como a nivel nacional.

Palabras clave: uso de tabaco; políticas públicas; estudiantes; conocimientos; actitudes y práctica en salud; hábito de fumar; control y fiscalización de productos derivados del tabaco

\section{Abstract}

Objective: To describe the knowledge and attitudes that the students of a University on cigarette smoking and the regulations that rule it, and how these regulations are applied in their practices. Methodology: Cross-section descriptive study, application of random stratified sampling. We developed the instrument taking some variables from the GATS and GYTS surveys. Results and Discussion: Women considered themselves passive smokers $(10 \%)$ and men, former smokers $(18.9 \%)$ The life prevalence was $53.3 \%$; prevalence during last month was $37.7 \%$ in males. $67.7 \%$ affirmed that the prevention measures of the Anti-tobacco law in Colombia have not had influence on the reduction of smoking in society. They recognized that there are measures to prevent smoking in the institution, but they consider that these should be strengthened to create a larger impact, both in the instruction and in the national level.

Keywords: tobacco use; public policies; students; knowledge; health attitudes and practice; smoking habit; control

\section{Resumo}

Objetivo: descrever os conhecimentos e atitudes dos estudantes de uma instituição universitária respeito do consumo de cigarro e as normas que regulamentam, e como estas normas são aplicadas nas suas práticas. Metodologia: estudo descritivo de corte transversal, aplicação de amostragem aleatório estratificado. Desenvolveu-se o instrumento tomando algumas variáveis dos inquéritos GATS e GYTS. Resultados e discussão: as mulheres consideraram-se fumadoras passivas (10\%) e os homens, exfumadores (18,9\%). A prevalência de vida foi de 53,3\%, a do último mês $37,7 \%$ em homens. $67,7 \%$ manifestou que as medidas de prevenção da lei colombiana antitabaco não influíram na diminuição do consumo na sociedade. Reconheceu-se que existem medidas de prevenção de consumo na instituição, mas considera-se que é necessário fortalece-las para gerar maior impacto tanto na mesma instituição como no nível nacional.

Palavras-chave: uso de tabaco; políticas públicas; alunos; conhecimentos; atitudes e prática em saúde; hábito de fumar; controle 


\section{Introducción}

El consumo de tabaco se ha convertido en una epidemia mundial prevenible, causada por el hombre, y es uno de los principales factores de riesgo de enfermedades crónicas como el cáncer, enfermedades pulmonares y cardiovasculares. Según la Organización Mundial de la Salud (oms), la epidemia de tabaquismo mata anualmente a más de cinco millones de personas, es decir, más que el VIH/SIDA, la tuberculosis y el paludismo juntos, y ha sido la causante de la muerte de uno de cada diez adultos. Asimismo, se estima que para el 2030 el consumo de tabaco podría cobrar la vida de más de ocho millones de personas anualmente (1). Las enfermedades y consecuencias derivadas del consumo de tabaco no se reflejan en la salud del fumador sino hasta años después de haber iniciado el consumo (2).

Hoy la tasa de mortalidad de los fumadores triplica a la de los no fumadores, lo que hace que un 30 a $35 \%$ de los primeros fallezcan antes de cumplir los 65 años. Con ello su vida se reduce en unos diez a quince años (3). Sin embargo, el humo de tabaco ajeno, conocido como el tabaquismo pasivo, también es causa de graves enfermedades cardiovasculares y respiratorias. De acuerdo con la oms, casi una tercera parte de los adultos se encuentran expuestos al humo de tabaco ajeno, de los cuales más de 600000 mueren anualmente por inhalar esta sustancia. Además de esto, se ha identificado que hay una probabilidad entre una y dos veces más de que un joven que esté expuesto al humo del tabaco en el hogar comience a fumar, que si no estuviera expuesto (4).

Una de las estrategias de control de consumo del tabaco más conocidas fue la declaración del Día Mundial sin Tabaco, celebrado el 31 de mayo de 1989, y actualmente el desarrollo del Convenio Marco para el Control del Tabaco (Смст), ratificado en el 2005 y en el cual se hizo un llamado a todos los países para la implementación de políticas públicas a través de medidas como "prohibiciones de la publicidad directa e indirecta del tabaco; aumento de impuestos y precios de productos de tabaco; creación de espacios sin humo en todos los lugares públicos y lugares de trabajo; y mensajes sanitarios bien visibles y claros en los paquetes de tabaco" (5).

En Colombia, el 27 de diciembre de 2006 a través de la Ley 1109 (6) se ratificó el смСт, y se inició con la reglamentación de la Resolución 1956 de 2008 (7) y la Ley 1335 de 2009 (Ley Antitabaco) (8), así como el Plan Nacional de Salud Pública 2007-2009 (9), en donde se contemplan aspectos como la prohibición del consumo de cigarrillo en las áreas cerradas de los lugares de trabajo, espacios públicos y el patrocinio de eventos deportivos, además de promoverse estrategias de información, educación, comunicación y asesoría para desestimular el hábito de fumar.

Para el municipio de Medellín, la Asociación Colombiana de la Salud realizó un estudio de consumo de tabaco en adolescentes en el año 2007, en el cual se destacó una prevalencia de consumo de tabaco en el último mes de $6,2 \%$, del cual $6,9 \%$ corresponde a hombres y $5,6 \%$ a mujeres. Al igual que otros estudios, en este se presentó un predominio de los hombres en el consumo de tabaco. Además, se observó que los adolescentes que no tienen grado de escolaridad están más expuestos a consumir esta sustancia; en ellos se encontró una prevalencia de consumo de casi el doble con respecto a los que tienen un grado de escolaridad (52,2\% versus $25 \%)(10)$.

Aunque las encuestas que ha construido la OMs han servido para estimar la prevalencia del consumo de tabaco y la exposición al humo que este genera $(11,12)$, se hace necesario examinar el problema desde otras perspectivas, es decir, entender el punto de 
vista de las comunidades realmente implicadas, los significados que dan al consumo de tabaco y cómo están conformadas por sujetos activos en la decisión sobre el consumo.

La investigación se enfocó en la población universitaria, ya que al ser parte de un proceso evolutivo a nivel académico, se puede indagar acerca de la percepción que tienen los estudiantes sobre el consumo de cigarrillo y qué factores consideran que pueden influir en su inicio o experimentación, lo cual incide en un aumento en el consumo en la población joven/universitaria (13). Han sido pocos los estudios que indagan sobre lo que piensan las personas acerca de la implementación de las políticas mencionadas previamente, por lo cual se hace necesario preguntarse por los conocimientos y las actitudes que tienen los estudiantes universitarios sobre el consumo de cigarrillo y las normas que lo regulan, con respecto a sus prácticas.

\section{Metodología}

Esta investigación es un estudio descriptivo de corte transversal, basado en fuente primaria, por medio de la encuesta. La población estuvo constituida por 7073 estudiantes matriculados en el 2013 en una institución universitaria pública de la ciudad de Medellín.

Se aplicó el muestreo aleatorio estratificado, el cual consiste en dividir la población en grupos o estratos con características diferentes entre sí, pero que en el interior de cada estrato guardan una similitud. Como estratos se tomaron las jornadas académicas diurna y nocturna. La muestra para la jornada diurna fue de 224 estudiantes y en la nocturna 140 .

Para controlar la elección de un mismo estudiante se usaron cinco filtros que permitieron tomar un horario por grupo, disminuyendo así la probabilidad de que el estudiante se en- cuentre en más de una ocasión. De acuerdo con los filtros utilizados, el número de grupos seleccionados fue de 982 .

Dentro de los grupos seleccionados se calculó la proporción que tiene la muestra con respecto a la población total, lo cual dio como resultado $5,15 \%$. Posteriormente, esta misma proporción se aplicó para determinar el número de grupos, y en este caso el resultado fue 52, entre los cuales se distribuyeron los 364 estudiantes.

Se identificaron 32 grupos para la jornada diurna y 20 para la nocturna, se seleccionaron siete estudiantes por grupo, los cuales fueron elegidos a través de muestreo aleatorio simple, mediante listado proporcionado por la institución.

El cuestionario se diseñó con base en la revisión de encuestas empleadas en otros estudios, como la Encuesta Mundial de Tabaquismo en Adolescentes (EMTA) y la Encuesta Global de Tabaquismo en Adultos (GATS), y se adaptó a la estructura de la encuesta de Conocimiento, Actitudes y prácticas (CAP). Fue sometido a dos pruebas piloto, con el fin evaluar aspectos metodológicos de validez, coherencia de las preguntas y tiempo de respuesta por parte de los encuestados. Se entregó consentimiento informado para aprobación y firma del estudiante. Asimismo, se siguió la Resolución 8430 de 1993, en la cual se afirma que "el sujeto de estudio, deberá prevalecer el criterio del respeto a su dignidad y la protección de sus derechos y su bienestar".

Se efectuó un análisis univariado y bivariado de las cincuenta variables relacionadas con los conocimientos, las actitudes, las prácticas del consumo de cigarrillo y las políticas que lo regulan. Las pruebas utilizadas para el análisis bivariado fueron Chi cuadrado y $\mathrm{T}$ de Student para determinar algunos factores asociados al consumo de cigarrillo, según correspondiera la naturaleza y la distribución de las variables, 337 con un nivel de significancia de $\mathrm{p}<0,05$. 


\section{Resultados}

El promedio de edad de los estudiantes fue de 23,9 años, con una desviación de 5,8 años. El coeficiente de variación entre las edades de las mujeres $(22,7 \%)$ y los hombres $(21 \%)$ indica que las edades de estos son heterogéneas. De los estudiantes encuestados, $54,7 \%$ eran mujeres, $61,2 \%$ pertenecían la jornada diurna y 72,3\% residían en Medellín, seguida de Bello con 13,4\%. Asimismo, el estrato socioeconómico que predominó fue el 3, con $49,7 \%$, seguido del $2(37,7 \%)$. Además, $45,0 \%$ de los encuestados tenían como nivel de estudio una tecnología. El semestre al que pertenecían, en su mayoría, era primero $(20,1 \%)$, seguido de quinto $(15,4 \%)$. En el aspecto laboral, $57,5 \%$ trabajan. Con respecto a estos tres últimos aspectos se encontró una relación con el consumo de cigarrillo $\left(\mathrm{Chi}^{2}: p<0,05\right)$.

\section{Categorías de consumidores según los estudiantes}

Para esta investigación se consideraron cuatro categorías relacionadas con el consumo o exposición al cigarrillo. Estas fueron: fumador habitual, fumador ocasional, fumador pasivo y exfumador.

Se destaca que más de la mitad $(52,7 \%)$ de los encuestados no se percibieron dentro de las opciones mencionadas anteriormente. Los investigadores consideraron que los jóvenes no percibían esta situación como un factor de riesgo para su salud. Además, los estudiantes, en un $14 \%$ se consideraron fumadores pasivos y exfumadores. Respecto al sexo, $18,9 \%$ de las mujeres se consideraron fumadoras pasivas. De manera similar, los hombres en $18,9 \%$ se consideraron exfumadores. Asimismo, se destaca que de los 53 estudiantes que se consideraron fumadores habituales y ocasionales, en promedio $71 \%$ eran hombres.

En cuanto a la edad, resalta el hecho de que los estudiantes entre 14 y 18 años se consideraran fumadores habituales, ocasionales y exfumadores en un $8,5 \%$, mientras que los de 18 a 22 años, en promedio el $15,7 \%$ se consideraron fumadores pasivos y exfumadores. Los de 22 a 25 años, en un $15,9 \%$ expresaron ser fumadores ocasionales y pasivos, y los mayores de 25 años eran exfumadores.

En cuanto a los fumadores habituales, 70,6\% eran hombres y $29,4 \%$ del total de fumadores habituales tenían entre 18 y 22 años. $\mathrm{Al}$ desagregar este grupo de edad por sexo, se destaca que son más las mujeres que se consideran fumadoras habituales $(60,0 \%)$ o fumadoras pasivas $(71,2 \%)$ (tabla 1$)$.

TABLA 1. Categoría de FUMAdor de los ESTUdiantes universitarios SEgún SEXo, 2013

\begin{tabular}{|c|c|c|c|c|c|c|}
\hline Categoría fumador & Mujeres & Porcentaje & Hombres & Porcentaje & Total & Porcentaje \\
\hline No responde & 9 & 4,6 & 3 & 1,9 & 12 & 3,4 \\
\hline Fumador habitual & 5 & 2,6 & 12 & 7,5 & 17 & 4,8 \\
\hline Fumador ocasional & 10 & 5,1 & 26 & 16,4 & 36 & 10,1 \\
\hline Fumador pasivo & 37 & 18,9 & 15 & 9,4 & 52 & 14,6 \\
\hline Exfumador & 21 & 10,7 & 30 & 18,9 & 51 & 14,4 \\
\hline Ninguna de las anteriores & 114 & 58,2 & 73 & 45,9 & 189 & 52,7 \\
\hline Total & 196 & 100,0 & 159 & 100,0 & 358 & 100,0 \\
\hline
\end{tabular}

Fuente: elaboración propia 


\section{Conocimiento de los estudiantes sobre el consumo de cigarrillo}

Con respecto a las normas que se aplican en el hogar en relación con el consumo de cigarrillo, 56,2\% de los encuestados que manifestaron que en su hogar existían normas eran mujeres. También se destaca que en los hogares donde se implementan normas con respecto al consumo de cigarrillo, $34,9 \%$ son estudiantes que tienen entre 18 y 22 años $(69,1 \%)$.

En cuanto a la condición laboral, 57,5\% de los encuestados trabajan. De estos, 53,4\% manifestaron que sus compañeros de trabajo fuman en espacios abiertos. Asimismo, un $51,0 \%$ afirmó que en su lugar de trabajo existen medidas de prevención de consumo de cigarrillo, siendo la más implementada la prohibición de consumo (19,9\%). De acuerdo con esto se encontró que el tener un trabajo, tener compañeros que fuman y disponibilidad de espacios para ello, tiene relación con que el estudiante fume ( $\mathrm{Chi}^{2}$ : $p<0,05)$.

Acerca de cómo se ha obtenido información sobre los efectos dañinos del cigarrillo, los estudiantes manifestaron en un $57,2 \%$ que han recibido información a través de los medios de comunicación. No se hallaron diferencias significativas por sexo. Sin embargo, se destaca que de los estudiantes que se declararon fumadores habituales, $33,3 \%$ no han recibido ayuda para dejar el cigarrillo y tienen entre 18 y 22 años. Asimismo, acerca de los mensajes de prevención, $85,4 \%$ de los encuestados han visto o escuchado mensajes sobre los efectos dañinos del cigarrillo a través de medios de comunicación, los cuales han sido más vistos por las mujeres $(48,7 \%)$.

En contraste con lo anterior, se sigue observando la emisión de mensajes que promueven el consumo de cigarrillo; $68 \%$ de los encuestados han visto este tipo de mensajes. Asimismo, se identificó en un $15 \%$ la distribución gratuita de cigarrillos a hombres.

$\mathrm{Al}$ indagar por la opción más dañina para consumir cigarrillo, las mujeres consideraron que inhalar el humo de otras personas $(63,5 \%)$ es más perjudicial para la salud. Esta misma opinión la dan los hombres con un $61,8 \%$. De acuerdo con los grupos de edad, se identificó esta misma opción en los estudiantes de 18 a 22 años $(33,0 \%)$.

Sobre las medidas que se implementan en la institución educativa sobre prevención del consumo de cigarrillo, $55,3 \%$ de los encuestados manifestaron que sí se implementaban medidas, sin presentarse diferencias por sexo. De los que declararon que sí había medidas, $39 \%$ tenían entre 18 y 22 años. De las medidas implementadas en la institución, resalto la restricción en lugares específicos con un $28,8 \%$, seguida de la prohibición de la venta en el interior de la institución con un $21,2 \%$. Esto fue identificado en más del $50 \%$ por las mujeres.

Acerca de lo que piensan los encuestados sobre quiénes fuman más, $50 \%$ manifestaron que no hay mucha diferencia entre hombres y mujeres; sin embargo, 29,9\% afirman que son más los hombres los que fuman que las mujeres; esta opinión es similar entre los encuestados por sexo.

Actitudes de los estudiantes sobre el consumo de cigarrillo

Entre los estudiantes encuestados se encontró que el principal motivo por el cual fumaron cigarrillo fue por curiosidad, manifestado principalmente por las mujeres con un $44,0 \%$, las cuales pertenecían al grupo de edad entre 18 y 22 años. Aunque esta fue la respuesta más frecuente, no fue la única variable que se relacionó con el consumo de 
cigarrillo. Factores como el querer parecer un adulto, la influencia de los amigos, los problemas personales, la publicidad y la soledad se relacionaron con el hábito de fumar $\left(\mathrm{Chi}^{2}: p=0,00\right)$.

Es notorio además que la circunstancia por la cual se siente más ganas de fumar es cuando se encuentran consumiendo bebidas alcohólicas, más en hombres $(53,8 \%)$ que en mujeres $(46,2 \%)$.

Se indagó si los estudiantes se han sentido incómodos en presencia de personas que fuman, y se encontró que el $81,8 \%$ se ha sentido así, con mayor incomodidad en las mujeres $(57,9 \%)$. Asimismo, la actitud que toman con respecto a las personas que fuman, es alejarse de ellas $(53,2 \%)$, con poca diferencia por sexo.

Con respecto a la prohibición de consumo de cigarrillo en lugares públicos, los encuestados declararon estar de acuerdo con esta prohibición en más del $70 \%$, igualmente más del $80 \%$ acepta que se debe prohibir el consumo de cigarrillo en el lugar de trabajo y en sitios cerrados como los restaurantes.
De igual manera, el 51,7\% está de acuerdo en la prohibición en lugares de expendio de licor (figura 1).

Acerca de las disposiciones sobre la prohibición de fumar cigarrillo, los estudiantes declararon estar de acuerdo en un 53\% con que el Estado intervenga en los comportamientos de las personas. Asimismo, 78,9\% están de acuerdo en que estas disposiciones buscan el cuidado de las personas y se deben respetar; además, 54,6\% consideran que las prohibiciones reducirían gastos en salud por enfermedades relacionadas con el consumo de cigarrillo.

Se indagó asimismo por la efectividad que ha tenido la implementación de la ley en Colombia sobre la prevención del consumo de cigarrillo, y se destaca que el 67,9\% de los encuestados opinaran que estas medidas no han influido en la disminución del consumo, opinión manifestada principalmente por las mujeres $(76,6 \%)$. Esta opinión es semejante con respecto a los estudiantes que fumaron en el último mes, los cuales indicaron en un $68,3 \%$ que estas medidas no han influido en la disminución del consumo. Consideran además que tienen un buen estado de salud $(32,4 \%)$.

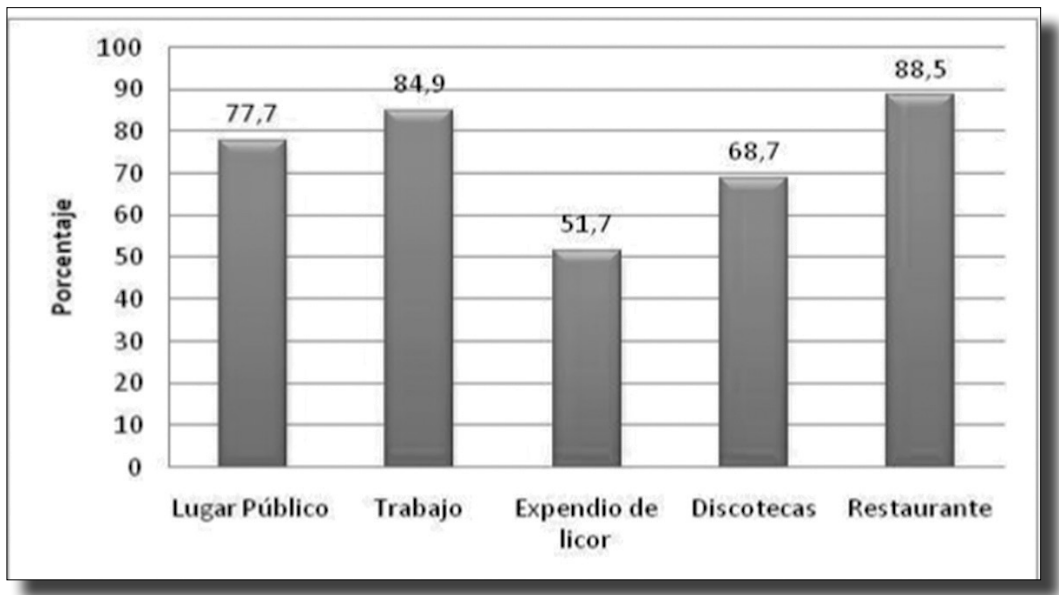

Figura 1. Sitios de PROHibición PARA FUMAR ACEPTAdos POR LOS ESTUdIANTES DE UNA INSTITUCiÓN UNIVERSITARIA, 2013

Fuente: elaboración propia 


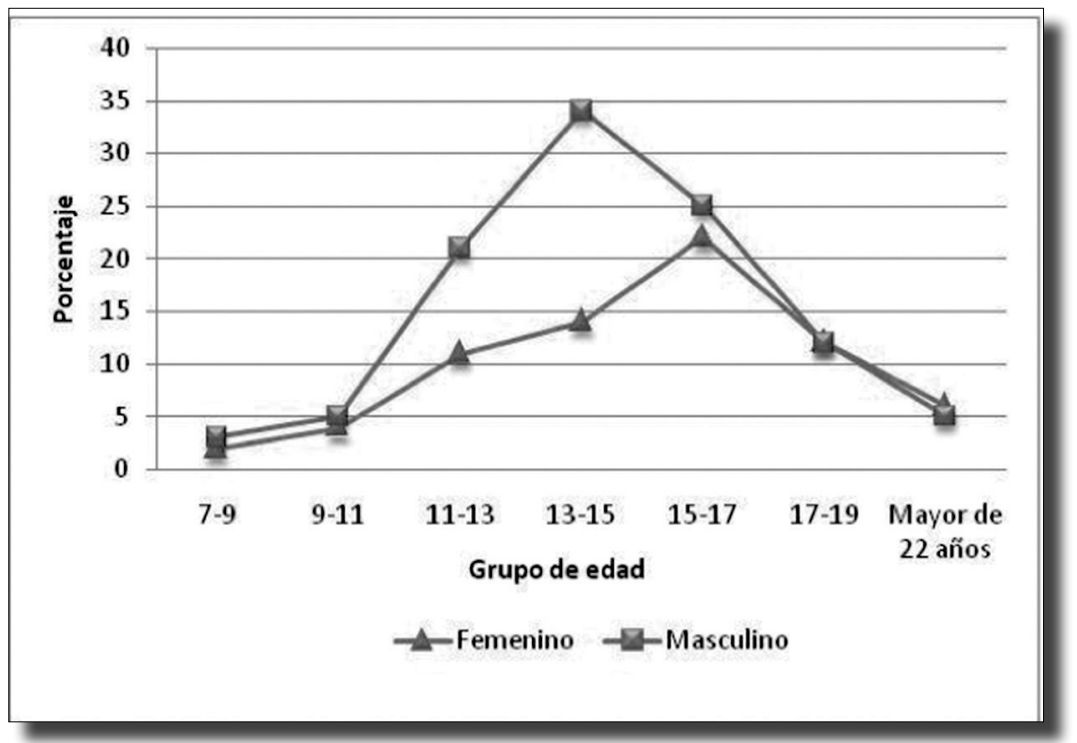

Figura 2. Edad de INICIO de CONSUMO de CIGARRILlo EN ESTUdiantes dE UNA INSTITUCión UNIVERSITARIA PÚBLICA, 2013

Fuente: elaboración propia

Finalmente, al preguntar a los encuestados si era difícil dejar de fumar después de haber iniciado el consumo, $55,1 \%$ de las mujeres y $41,7 \%$ de los hombres respondieron que sí, y para los que fumaron en el último mes no hubo diferencias en la opinión sobre la dificultad de dejar de fumar.

\section{Prácticas de consumo de cigarrillo}

$\mathrm{Al}$ indagar por la prevalencia de vida, 53,3\% de los encuestados manifestaron haber fumado alguna vez en la vida. En la población masculina se encontró que el $71,1 \%$ ha fumado alguna vez, mientras que en las mujeres hubo consumo del cigarrillo alguna vez en un $39,6 \%$. Para los grupos de edad, es más alta la prevalencia de las mujeres que fuman entre 19 y 22 años, mientras que en los hombres el grupo de edad se amplía hasta los 25 años. Existe entonces una relación entre género y fumar $\left(\mathrm{Chi}^{2}: p=0,00\right)$.

Asimismo, de los estudiantes que manifestaron haber fumado alguna vez en su vida, en promedio iniciaron su consumo a los 14 años (DE $=4,9)$; los hombres inician a los 13 años y las mujeres a los 15 (figura 2). Sin embargo, se destaca que no se encontraron diferencias en las medias de la edad de inicio de fumar de hombres y mujeres (T de Student: $p=0,069$ ).

Realizando una comparación por jornadas con respecto a los estudiantes que alguna vez fumaron en su vida, se encontró que 51,6\% pertenecían a la jornada diurna y 55,8\% a la nocturna. Se destaca que en la nocturna fueron más los hombres los que manifestaron haber consumido alguna vez $(72,7 \%)$.

Con respecto al grupo de edad, se identificó que los estudiantes de la jornada diurna que alguna vez fumaron tenían entre 12 y 18 años $(45,9 \%)$, y en la nocturna $31,1 \%$ tenían entre 22 y 25 años.

En cuanto a la prevalencia del último mes, ${ }^{1}$ se encontró que $37,7 \%$ de los que alguna

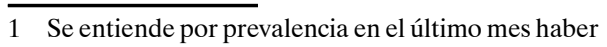
consumido al menos un cigarrillo en los últimos 
Tabla 2. Consumo de Cigarrillos al día en El Último Mes POR los ESTUdiantes Universitarios, SEgún SEXo Y JORNADA ACADÉMICA

\begin{tabular}{|l|c|c|c|c|c|c|c|c|}
\hline \multirow{2}{*}{$\begin{array}{l}\text { Consumo cigarrillo } \\
\text { último mes }\end{array}$} & \multicolumn{4}{|c|}{ Jornada diurna } & \multicolumn{4}{c|}{ Jornada nocturna } \\
\cline { 2 - 10 } & $\mathbf{n}$ & Porcentaje & N & Porcentaje & n & Porcentaje & n & Porcentaje \\
\hline Menos de 10 & 14 & 27,5 & 10 & 17,5 & 2 & 10,5 & 11 & 19,6 \\
\hline Más de 10 & 7 & 13,7 & 6 & 10,5 & 0 & 0 & 12 & 21,4 \\
\hline Más de 20 & 0 & 0 & 2 & 3,5 & 2 & 10,5 & 3 & 5,4 \\
\hline $\begin{array}{l}\text { No fumó en el } \\
\text { último mes }\end{array}$ & 30 & 58,8 & 39 & 68,4 & 15 & 78,9 & 30 & 53,6 \\
\hline Total & $\mathbf{5 1}$ & $\mathbf{1 0 0}$ & $\mathbf{5 7}$ & $\mathbf{1 0 0}$ & $\mathbf{1 9}$ & $\mathbf{1 0 0}$ & $\mathbf{5 6}$ & $\mathbf{1 0 0}$ \\
\hline
\end{tabular}

Fuente: elaboración propia

vez fumaron en su vida, lo hicieron en el último mes, consumo que se presentó más en los hombres en un $63,8 \%$. Estos además declararon en un $20,2 \%$ que han consumido menos de 10 cigarrillos al día.

Al hacer la misma comparación por sexo y jornada, se encontró que $84 \%$ de las mujeres que fumaron en el último mes pertenecían a la jornada diurna. En cambio, los hombres en un $59 \%$ fumaron más en el último mes y pertenecían a la jornada nocturna (tabla 2).

También fue de interés indagar si el ingreso a la institución educativa influyó en el estudiante para el inicio del consumo; lo hallado indica que $85,5 \%$ de los que fumaron en el último mes manifestaron no haber sido influidos para consumir cigarrillo al momento de ingresar a la institución. Esto lo manifestaron más los hombres $(64,4 \%)$, sin embargo, existe una relación entre el ingreso a la institución y el inicio del consumo $(p=0,017)$.

Se destaca que de los estudiantes que fumaron en el último mes solo $18 \%$ manifestaron fumar más durante las primeras horas del día, lo que en mayor proporción dijeron hacerlo mujeres $(63,6 \%)$. Adicionalmente,

treinta días. Para los fines de este análisis se considerará como "fumadores" a estas personas. cuando se indagó si alguna vez han recibido ayuda para dejar de fumar, solo $37,7 \%$ manifestaron no haber recibido ayuda. Sin embargo, de los estudiantes que fumaron alguna vez, $62,6 \%$ manifestaron que han intentado dejar de fumar por sí solos y lograron dejarlo, principalmente las mujeres $(70,8 \%)$. No lo ha intentado el $20,1 \%$ y solo un $10,8 \%$ lo ha retomado. De acuerdo con la edad, es de notar que los que lo intentaron y lo dejaron tenían entre 18 y 22 años $(54,2 \%)$.

La razón principal por la que los estudiantes dejaron el consumo de cigarrillo fue por razones de salud $(69,0 \%)$, en tanto que quienes retomaron el consumo sostuvieron que no tenían fuerza de voluntad $(46,7 \%)$, especialmente los hombres $(71,4 \%)$. Además, se indagó si el haber dejado de fumar fue reemplazado por otro hábito, y el 42,5\% de los estudiantes dijo no haberlo reemplazado con nada, seguidos de un $28,7 \%$ que lo reemplazó por el deporte, especialmente los hombres $(68 \%)$.

Al preguntar si el entorno en el que se desenvuelve el estudiante es libre de humo de cigarrillo, se encontró que tanto hombres como mujeres, en más del $30 \%$, se encuentran rodeados de más de dos personas que fuman en su ambiente. Por último, se preguntó a los estudiantes si se han sentido influidos por 
la publicidad del consumo de cigarrillo, y el 13,6\% mencionó sentirse así. Este porcentaje puede considerarse bajo, sin embargo, puede detectarse que la publicidad aún tiene fuerza en la influencia, por lo cual debe tenerse en cuenta en la construcción de políticas de reducción de consumo de cigarrillo.

\section{Discusión}

La encuesta CAP sobre tabaquismo aplicada a la población universitaria se realizó como una estrategia para profundizar el conocimiento sobre este tema, considerado problema de salud pública, y proporcionar información clave a fin de que la institución tome decisiones tempranas y fortalezca las medidas de prevención y control.

En la muestra de la investigación predominó el sexo femenino $(54,7 \%)$ y el grupo de edad de 18 a 22 años. Esta situación fue similar en otros estudios (14), en donde más del $50 \%$ de los estudiantes eran mujeres y, asimismo, su edad oscilaba entre 17 y 24 años.

Con relación a los conocimientos que los estudiantes tenían sobre el consumo de cigarrillo, especialmente sobre cómo se consideraban ellos, con independencia de si fumaban o no, se destacó que más de la mitad declararon ser no fumadores, fumadores pasivos o exfumadores. Cabe pensar que los estudiantes no se han relacionado con personas que hayan consumido o quizás nunca se habían preguntado sobre esta cuestión.

Se resalta que menos del $60 \%$ de los estudiantes han recibido información sobre los efectos dañinos del cigarrillo a través de medios de comunicación. En un estudio realizado en Paraguay casi el $90 \%$ de los estudiantes conocían los efectos dañinos, información que fue proporcionada por el colegio, seguido de la universidad. Asimismo, tanto hombres como mujeres declararon que es más dañino inhalar el humo de otras personas que fumar (15).

A pesar de los mensajes de prevención del consumo que se transmiten por varios medios, se resalta que aún se siguen viendo mensajes que promueven el consumo. Esto fue más captado por los hombres que por las mujeres, lo cual puede incidir en que aquellos sigan consumiendo más que estas.

Con respecto a las medidas de prevención en la institución universitaria, casi el 50\% conocía que estas se implementaban, a diferencia del trabajo realizado en Paraguay en donde los estudiantes desconocían la existencia de normas. No obstante, este último coincide con la presente investigación en que las medidas implementadas fueron la restricción del consumo en lugares específicos y la prohibición de venta en el interior de la institución (13). Sin embargo, a pesar de la existencia de estas medidas, más de la mitad de los estudiantes estuvieron de acuerdo en que no han sido efectivas, ni han influido en la disminución del consumo.

Finalmente, sobre las diferencias de género y consumo de tabaco, se destaca que desde finales del siglo XIX se comenzó a comercializar el tabaco y este fue consumido por hombres de la clase alta en países desarrollados y por mujeres consideradas indecentes. Sin embargo, a partir del siglo $\mathrm{xx}$ las mujeres desafiaron las normas establecidas y aumentaron su consumo de tabaco, con lo que empezaron a estabilizarse las prevalencias de este entre hombres y mujeres, situación que se sigue presentando en la actualidad (16). En la investigación, al preguntarse sobre quiénes fumaban más entre los dos sexos, más o menos el $50 \%$ de los estudiantes estuvieron de acuerdo en que ya no había mucha diferencia de consumo entre hombres y mujeres. Esto se corrobora en estudios 
realizado en España en donde el consumo de cigarrillo no tiene diferencias significativas por sexo (17).

Varios estudios corroboran que la prevalencia de consumo de cigarrillo alguna vez en la vida es más alta en los hombres en más del $60 \%$ (14); esto se explicaría por la circunstancia de tener un amigo fumador, lo cual ejerce una gran influencia en el consumo de tabaco, como lo reflejan numerosos estudios (18). En cuanto a la edad, en la presente investigación $34 \%$ de los estudiantes que fumaron alguna vez tenían entre 19 y 22 años, lo cual indica que cada vez es más temprana la edad en que llegan a consumir por primera vez; sin embargo, en un estudio realizado en Perú entre estudiantes universitarios, el consumo era mayor entre los que tenían entre 27 y 30 años (19).

En relación con la edad de inicio del consumo, en promedio, los estudiantes que manifestaron haber fumado alguna vez iniciaron su consumo a los 14 años, lo que hace pensar que su consumo se presentaba antes de iniciar en la universidad; esto se corrobora cuando responden que el ingreso a la institución no influyó en el inicio del consumo. Para los hombres se presentó el inicio del consumo a los 13 años, mientras que el inicio en las mujeres fue más tardío, a los 15 años (figura 2). Los estudios anteriormente mencionados $(15,19)$ también presentan una edad de inicio antes de los 20 años, lo que puede indicar que es más probable que se siga consumiendo en la edad adulta y que sea menos probable que se deje el hábito.

Además, al indagar sobre el principal motivo que tuvieron los estudiantes al momento de probar el cigarrillo, estos manifestaron que lo habían probado por curiosidad, siendo esta una de las causas para que se pruebe cigarrillo por primera vez. En estudios anteriores 344 se ha identificado esta razón como un factor de riesgo para iniciarse en el consumo (20).
En cuanto a la prevalencia del último mes, $61,0 \%$ de los que alguna vez fumaron en su vida, no lo hicieron en el último mes, seguido de un 19,8\% que manifestó haber fumado menos de diez cigarrillos, sin presentarse diferencias significativas entre sexo. Esto podría indicar que aun cuando los estudiantes consumieron alguna vez, no tomaron como hábito el consumo y solo lo hicieron por curiosidad.

En este estudio se observa, así como en otros, que la variable de ingesta de alcohol está asociada al consumo de cigarrillo (21). Fumar y beber alcohol son dos actividades fuertemente relacionadas, y el aumento en el consumo de bebidas alcohólicas evoluciona de forma paralela al consumo de cigarrillo.

En este estudio se pretendió comparar el consumo de cigarrillo entre los estudiantes de las jornadas diurna y nocturna, intentando encontrar hallazgos significativos en las jornadas; se pensaba que en la jornada nocturna habría más estudiantes que consumieran cigarrillo debido a factores como la edad, el trabajo o el ambiente. Sin embargo, se encontró que son más los estudiantes de la jornada diurna los que consumen. Asimismo, entre las mujeres se reporta un consumo de menos de diez cigarrillos al día. Y en la jornada nocturna, son más los hombres los que consumen en promedio entre 11 y 20 cigarrillos al día. No obstante, en un estudio realizado en Villavicencio se encontró una prevalencia ligeramente superior en estudiantes que estudiaban carreras nocturnas (18).

Es necesario destacar que más del $50 \%$ de los estudiantes no han recibido ayuda para dejar de fumar. Esto indica que resultaría oportuno implementar programas de apoyo para aquellos estudiantes que desean dejar de fumar. A pesar de esto se identificó que los estudiantes dejaron el cigarrillo por sí so- 
los por razones de salud (22) y otros lo retomaron porque no tenían fuerza de voluntad.

No se debe ignorar a aquellos que sin consumir se ven afectados por el humo del cigarrillo. En diversos estudios se determinó que las principales enfermedades crónicas están asociadas con el tabaquismo y otros factores causales, algunas de estas enfermedades suelen manifestarse solo después de un periodo latente considerable, que representa el tiempo necesario para que el daño sea suficiente. Es el caso del cáncer de pulmón, cuya tasa de incidencia aumenta luego de 20 años de fumar activamente (23). La enfermedad pulmonar obstructiva crónica (EPOC), la cual altera la respiración normal y es potencialmente mortal, es una afección relacionada principalmente con el humo del tabaco; sin embargo, en nuevas investigaciones se ha determinado que no solo se presentan casos en personas fumadoras activas, sino también en fumadores pasivos. Se prevé que en ausencia de intervenciones para reducir los riesgos, y en particular la exposición al humo del tabaco, las muertes por EPOC aumenten en más de un $30 \%$ en los próximos 10 años (24).

Desde el punto de vista de los estudiantes, $67,9 \%$ manifestaron que las medidas implementadas por la ley para prevenir el consumo no han sido suficientemente efectivas como se esperaba. Pero cabe mencionar que estas afirmaciones no cuentan con estudios base para determinar que no se esté cumpliendo a cabalidad o en parte la ley antitabaco colombiana. En una investigación realizada con estudiantes universitarios en España, estos consideran que la ley antitabaco de ese país sí se cumple y que se llevan a cabo las medidas implementadas; resaltan además que han sido beneficiosas socialmente. A partir de dicha ley ha dejado de fumar el 16,5\% y el $35,5 \%$ se ha planteado abandonarlo. El $24,6 \%$ no la respeta (25).
Se resaltan los esfuerzos realizados en la institución universitaria para cumplir lo estipulado en la ley, basados específicamente en el convenio marco el cual determina un amplio acceso a programas integrales y eficaces de educación y concientización de la sociedad sobre los riesgos que acarrean para la salud el consumo de tabaco y la exposición al humo de tabaco (1). Con la implementación de campañas internas, de sitios libres de humo, la prohibición de venta al menudeo en el interior de la institución, y más actividades, se pretende reducir al máximo los daños ocasionados por el cigarrillo, siendo la única causa prevenible de muerte y enfermedades.

\section{Conclusiones}

La población que más sobresalió al momento de aplicar la encuesta fueron las mujeres, y fueron más los hombres los que se consideraron ser fumadores habituales, en tanto que las mujeres manifestaron ser fumadoras pasivas.

Los estudiantes que han sido considerados fumadores habituales, recibieron información sobre los efectos dañinos del humo del cigarrillo, siendo los medios de comunicación (televisión, radio, periódicos, revistar, internet) los más usados para transmitir esta información; igualmente, confirman haber escuchado, visto información sobre medidas de prevención y prohibición del consumo en lugares públicos, el lugar de trabajo y confirman tener conocimiento sobre las campañas implementadas en la institución educativa.

Diversos factores se relacionan con que el estudiante inicie a temprana edad, como lo es la curiosidad, la influencia de los amigos, los problemas personales, la publicidad y la soledad.

La edad de inicio para fumar no es diferente entre hombres y mujeres. Las mujeres están 
cerrando la brecha y se ubican a la par con los hombres al momento de empezar a fumar.

\section{Al entrar al ambiente de una institución educativa, el estudiante comienza a expe- rimentar nuevas vivencias, entre ellas el consumo de cigarrillo, aunado a los factores mencionados previamente.}

La falta de fortalecimiento de las campañas sobre el consumo de cigarrillo dentro de la institución incide en el desconocimiento de las normas establecidas fuera y dentro de esta, y en cierta forma persiste la falta de conciencia sobre el deterioro de la salud, lo que conlleva el incremento de enfermedades crónicas y el aumento de costos en la salud.

\section{Agradecimientos}

A la institución universitaria Tecnológico de Antioquia por brindarnos el espacio para llevar a cabo la investigación. A la profesora Difariney González por su apoyo en la elección de la metodología.

\section{Referencias bibliográficas}

1. Organización Mundial de la Salud (OMs). Iniciativa Liberarse del Tabaco. ¿Por qué el tabaco es una prioridad de salud pública? Ginebra: oms; 2011 [Internet] [acceso: 11 de octubre del 2012]. Disponible en: http://www.who.int/tobacco/health_priority/ es/index.html.

2. Organización Mundial de la Salud (oms). 10 datos sobre la epidemia del tabaquismo y el control mundial del tabaco. Washington: oms; 2011 [Internet] [acceso: 5 de diciembre del 2012]. Disponible: http://www.who.int/features/factfiles/tobacco_epidemic/es/index.html

3. Organización Mundial de la Salud (OMS). MPOWER: un plan de medidas para hacer retroceder la epidemia de tabaquismo. Ginebra: oms; 2008 [Internet] [acceso 10 de octubre de 2012]. Disponible en: http://www.who.int/tobacco/mpower/mpower_spanish.pdf.

4. Organización Mundial de la Salud (oms). 10 datos sobre el tabaquismo pasivo. Washington: oMs; 2009 [Internet] [acceso: 12 de agosto del 2012]. Dis- ponible en: http://www.who.int/features/factfiles/ tobacco/es/index.html.

5. Asamblea Mundial de la Salud. Convenio Marco de la oms para el control del Tabaco. Ginebra: La Asamblea; 2003 [Internet] [acceso: 7 de noviembre del 2012]. Disponible en: http://www.paho.org/ Spanish/DD/PuB/sa56r1.pdf.

6. República de Colombia. Ley 1109, 27 de diciembre de 2006: por medio de la cual se aprueba el "Convenio Marco de la oms para el control del tabaco", hecho en Ginebra, el veintiuno (21) de mayo de dos mil tres (2003); Bogotá: El Congreso; 2006.

7. República de Colombia. Resolución 1956, 30 de mayo de 2008: por la cual se adoptan medidas en relación con el consumo de cigarrillo o de tabaco. Bogotá: El Ministerio; 2008.

8. República de Colombia. Ley 1335 del 21 de julio de 2009: por la cual se previenen daños a la salud de los menores de edad, la población no fumadora y se estipulan políticas públicas para la prevención del consumo del tabaco y el abandono de la dependencia del tabaco del fumador y sus derivados en la población colombiana. Bogotá: El Congreso: 2009.

9. Organización Mundial de la Salud (OMs). Plan Nacional de Salud Pública 2007-2010. Bogotá: OMS; 2007 [Internet] [acceso: 2 de septiembre del 2012]. Disponible en: http://www.minsalud.gov.co/salud/ Paginas/SaludP\%C3\%BAblica.aspx

10. Duque L, Montoya N, Toro J, Restrepo A. Consumo de tabaco en adolescentes en Medellín, 2007. Medellín: Asociación Colombiana de la Salud; 2008.

11. Organización Panamericana de la Salud (ops), Instituto Nacional de Salud Pública (INS). Encuesta Global de Tabaquismo en Adultos. México 2009. México: OPS, INS; 2010.

12. Wiesner C, Peñaranda D. Encuesta mundial de tabaquismo en jóvenes. Reporte Bogotá, Colombia. Revista Colombiana de Cancerología. 2002; $6(4): 5-14$.

13. Villar E, Viñas F, Ferrer J, Caparros B, Pérez I, Cornella M. Dimensiones psicopatológicas asociadas al consumo de tabaco en población universitaria. Anal. Psicol. 2004; 20 (1): 33-46.

14. Montoya E, Cunningham J, Brands B, Strike C, Miotto MG. Consumo percibido y uso de drogas lícitas e ilícitas en estudiantes universitarios en la ciudad de Medellín, Colombia. Rev Latino-Am Enfermagem [serie en Internet] 2009; 17 (Especial) [acceso: 2 de octubre del 2012]. Disponible en: http://www.scielo.br/scielo.php?script = sci_arttext \&pid $=$ S0104-11692009000700020

15. Morel JC. Conocimientos, actitudes y prácticas sobre el tabaquismo en estudiantes de Enfermería y Obstetricia del Instituto "Dr. Andrés Barbero"Año-2007. Mem Inst Investig Cienc Salud [serie en Internet]. 2008; 4 (2) [acceso: 12 de septiembre del 2012]. Disponible en: http://scielo.iics.una.py/pdf/ iics/v6n2/v6n2a07.pdf.

16. Laespada MT. Diferencias de género y consumo de tabaco. X Jornadas Sedet: Tabaco y desigualdad, 5 de mayo del 2011, Bilbao, España: Sedet; 2011 [Internet] [acceso: 10 de septiembre del 2012]. Dis- 
ponible en: http://www.sedet.es/webcms/usuario/ documentos/XJornadas-MTeresa-Laespada.pdf

17. Delgado B, Bautista R, Inglés C, Espada JMT. Diferencias de género en el consumo de alcohol y tabaco de estudiantes de educación secundaria obligatoria. Salud y drogas [serie en Internet]. 2005; 5 (2) [acceso: 5 de noviembre del 2012]. Disponible en: http://www.haaj.org/sites/default/files/DIFERENCIAS \%20DE\%20G\%C3\%89NERO.pdf

18. García-Baquero MR, Tobón-Borrero LM. Consumo de sustancias psicoactivas en estudiantes de primer semestre de las universidades del municipio de Villavicencio 2009. Rev Salud Hist Sanid [serie en Internet]. 2011; 6 (1) [acceso 05 de septiembre de 2012] Disponible en:

19. http://virtual.uptc.edu.co/revistas/index.php/shs/ article/viewFile/2139/2042.

20. León D, Ferreira PSP. Conocimientos y practicas sobre el consumo de tabaco en estudiantes de pregrado de farmacia, Lima, Perú. Rev Latino-Am Enfermagem [serie en Internet]. 2010; 18 (especial) [acceso: 12 de octubre del 2012]. Disponible en: http://www.scielo.br/scielo.php?script =sci_arttext \&pid=S0104-11692010000700014
21. Castaño G. Módulo educativo: consumo de drogas en universitarios estrategias de actuación. Medellín Funlam, FIUC; 2008 [Internet] [acceso: 10 de octubre del 2012]. Disponible en: http://comunidad.udistrital.edu.co/udesigual/files/2013/02/kit-educativo-deprevencion.pdf.

22. Caballero HA, González B, Pinilla JPB. Factores predictores del inicio y consolidación del consumo de tabaco en adolescentes. Gac Sanit. 2005; 19 (6): 444-7.

23. Argentina. Programa Nacional para el Control del Tabaco, Ministerio de Salud y Ambiente. Encuesta de tabaquismo en grandes ciudades de Argentina, 2004. Argentina: El Ministerio; 2005.

24. Samet J. Los riesgos del tabaquismo activo y pasivo. Salud Publica Mex. 2002; 44: 144-60.

25. Organización Mundial de la Salud (oms). Enfermedad pulmonar obstructiva crónica (EPOC). Washington: oms; 2012 [Internet] [acceso: 3 de marzo del 2013]. Disponible en: http://www.who. int/mediacentre/factsheets/fs315/es/.

26. Isla P, Moncho JML. Percepción de los estudiantes universitarios sobre la ley de medidas sanitarias frente al tabaquismo. Rev Enferm. 2010; 33 (78): 7-24. 